\title{
Next-Nearest-Neighbor Hopping in the Falicov-Kimball Model
}

\author{
K. CZajKa And M.M. MAŚKA \\ Institute of Physics, University of Silesia \\ Uniwersytecka 4, 40-007 Katowice, Poland
}

\begin{abstract}
Results of Monte Carlo simulations for the spinless Falicov-Kimball model with the next-nearest-neighbor hopping are presented. We find the critical value of the next-nearest-neighbor hopping integral, below which the low temperature configuration of the localized particles is the same as in the presence of only the nearest-neighbor hopping. Beyond this critical value the localized particles form horizontal or vertical stripes.
\end{abstract}

PACS numbers: 71.10.--w, 71.10.Fd

\section{Introduction}

The spinless Falicov-Kimball (FK) model [1] is a simple quantum model that describes itinerant electrons interacting with classical localized particles. Despite the simplicity, the FK model exhibits a rich phase diagram [2]. It has been rigorously proved that for dimensions $D \geq 2$ the system has a phase transition from a homogeneous high-temperature phase to a phase, in which the classical particles form a checkerboard pattern $[3,4]$. This property holds for any bipartite lattice and the FK model has been analyzed mostly on square or (hyper)cubic lattices, which fulfill this requirement. Much less is known about the properties of the FK model on non-bipartite lattices. The main question that arises in connection with non-bipartite lattices concerns the nature of the ground state, whether or not there is a long range order at low temperatures. The answer to this question depends, of course, on the topology and geometry of the lattice. In Ref. [5] we have shown that the FK model on a triangular lattice exhibits a finite-temperature phase transition, at least apart from the strong interaction limit, where it maps itself on the triangular Ising antiferromagnet that is known to exhibit a phase transition at $T=0[6]$.

In this paper we analyze the role of the next-nearest-neighbor hopping in the FK model on the square lattice. In particular, we demonstrate how the checkerboard ground state is modified when the next-nearest-neighbor hopping increases and how the critical temperature depends on its amplitude. 


\section{Model and method}

The Hamiltonian of the spinless FK model is given by

$$
\mathcal{H}=\sum_{i j} t_{i j} c_{i}^{\dagger} c_{j}+U \sum_{i} n_{i} w_{i} .
$$

Here, $n_{i}=c_{i}^{\dagger} c_{i}, w_{i}$ is equal to 0 or 1 , according to whether the site $i$ is occupied or unoccupied by a massive particle. The hopping integral $t_{i j}$ is equal to $-t$ when $i$ and $j$ are nearest neighboring lattice sites, $-t^{\prime}$ for next nearest neighbors, and zero otherwise. Without the $t^{\prime}$ term this model has been investigated by means of various methods [7]. However, up to our best knowledge, the influence of the next-nearest-neighbor hopping has been taken into account only in Ref. [8], where the FK model has been studied in perturbative regime, where $U \gg t, t^{\prime}$. In this paper we present results obtained with the help of a variant of Monte Carlo method that is suitable for a system with both classical and fermionic degrees of freedom. The details are described in Ref. [9]. The simulations were performed for $20 \times 20$ clusters with periodic boundary conditions. The concentrations of both the classical particles $\left(N_{i} / N\right)$ and the electrons $\left(N_{\mathrm{e}} / N\right)$ is equal to 0.5 .

\section{Results}

It is known that for $N_{i} / N=N_{\mathrm{e}} / N=0.5$ the classical particles form a checkerboard pattern in the ground state on a square lattice. This long-range order survives also up to some finite critical temperature, at which the system undergoes a phase transition to a homogeneous high-temperature phase. One may expect that the low-temperature checkerboard order minimize the free energy also in the presence of small next-nearest-neighbor hopping. The question arises what would be the actual low-temperature state for relatively large $t^{\prime}$ ? To answer this question let us take into consideration the limiting case of a very strong next-nearest-neighbor hopping $t^{\prime} \gg t$. In this limit the square lattice breaks up into two interpenetration sublattices with the lattice constant $\sqrt{2}$ times larger than the original one and with the axes rotated by 45 degrees. Since there is no intersite interactions neither between electrons nor between massive particles, and the next-nearest-neighbor hopping does not move electrons between sublattices, the sublattices are completely independent. As a result at low temperature in both these sublattices massive particles form the checkerboard pattern. Each of these subsystems is twofold degenerated (massive particles can be shifted into the empty sites). As a result of the degeneracy the massive particles in the whole system can form either horizontal or vertical stripes (see Fig. 1).

In order to investigate the regime of intermediate next-nearest-neighbor hopping let us recall that the FK model in the strong Coulomb interaction limit maps onto the antiferromagnetic Ising model. The Ising model on the square lattice with nearest-neighbor $(J)$ and next-nearest-neighbor $\left(J^{\prime}\right)$ couplings possesses two different ground states, depending on the $J^{\prime} / J$ ratio: for $J^{\prime} / J<0.5$ the ground state 


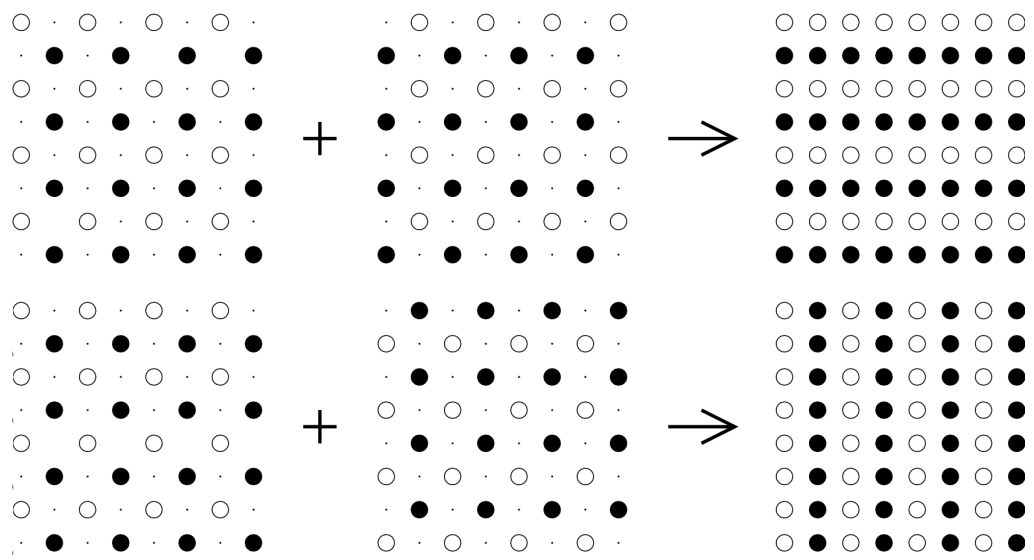

Fig. 1. Two possible low-temperature configurations of localized particles in the $t^{\prime} \gg t$ limit. Open circles represent empty sites within a given sublattice, whereas the filled ones indicate positions of the massive particles.

is the simple antiferromagnet, whereas for $J^{\prime} / J>0.5$ the system minimizes the energy by ordering in alternate ferromagnetic rows of opposite spins [10,11]. Such spin configurations ("superantiferromagnetic") correspond to the massive particle configurations of the FK model presented in Fig. 1. Since the ratio $J^{\prime} / J$ of Ising nearest-neighbor and next-nearest-neighbor interactions is equal to $\left(t^{\prime} / t\right)^{2}$ in the corresponding FK model, the threshold value of $t^{\prime}$ is given by $t^{\prime} / t \simeq 0.71$. This result holds in the limit $U \rightarrow \infty$. For weaker interaction this threshold ratio can be determined from a comparison of the ground state energy in both the checkerboard and stripe configurations.

In order to illustrate the transition from the checkerboard low- $t^{\prime}$ regime to the stripe high $-t^{\prime}$ one, we have carried out appropriate Monte Carlo simulations. Figure 2 shows "snapshots" of the system for various ratios $t^{\prime} / t$. In accordance with theoretical predictions for very small $t^{\prime}$ localized particles form the checkerboard pattern, the same as in the absence of the next-nearest-neighbor hopping (Fig. 2A). With increasing $t^{\prime}$ the critical temperature decreases and defects occur in the checkerboard pattern (Fig. 2B). The next configuration (Fig. 2C) corresponds to $t^{\prime}$ above the critical value, i.e., to the regime, where stripe configurations minimize the energy. However, due to the fourfold degeneracy of the ground state the overall configuration consists of segments of stripes. With further increase in the ratio $t^{\prime} / t$ (Figs. 2D and 2E) the localized particles arrange themselves into stripes. The imperfectness of the pattern is connected with two effects. First, the degeneracy of the ground state allows the system to choose one of them in one region of the cluster and another one in other region. In such a case phase boundary lines are inevitable. The second effect is connected with thermal fluctuations. When $t^{\prime}$ increases with fixed values of $t$ and $U$, the effective interaction strength, i.e., the interaction potential compared to the kinetic energy, decreases. As a result the 
(A)



Fig. 2. Examples of low-temperature configurations of localized particles for various values of the ratio $t^{\prime} / t$ : (A) 0.1 , (B) 0.4, (C) 0.8, (D) 1.1, (E) 1.4. All the configurations have been obtained for $U=t$ at temperature $k_{\mathrm{B}} T=0.02 t$.

critical temperature decreases as well and thermal fluctuations destroy the stripe pattern.

The presence of the next-nearest-neighbor hopping obviously modifies the critical temperature, which is connected with the frustration present in the system. With increasing $t^{\prime}$ the critical temperature decreases, going to zero for some critical value of $t^{\prime *}$. This value is equal to $\sqrt{2} / 2$ in $U / t \rightarrow \infty$ limit. For finite interaction $(U<\infty) t^{\prime *}$ always lies between $0.71 t$ and $0.8 t$. At this point there is no long range order at any nonzero temperature. For $t^{\prime}>t^{\prime *}$ the critical temperature increases with increasing $t^{\prime}$. In this regime the critical temperature is the temperature of the transition from the stripe configuration to the disordered state. Eventually, for $t^{\prime} / t \gg 1$ the critical temperature starts to decrease with increasing $t^{\prime}$, which is connected with increasing band width, much larger than the interaction potential.

\section{Summary}

We have demonstrated how properties of the Falicov-Kimball model on the square lattice are affected by the presence of the next-nearest-neighbor hopping. In particular, we have shown how the low-temperature configurations of the localized particles depend on $t^{\prime} / t$ and have discussed the critical temperature as a function of the next-nearest-neighbor hopping amplitude. 


\section{References}

[1] L.M. Falicov, J.C. Kimball, Phys. Rev. Lett. 22, 997 (1969).

[2] J.K. Freericks, Phys. Rev. B 47, 9263 (1993); 48, 14797 (1993).

[3] T. Kennedy, E.H. Lieb, Physica A 138, 320 (1986).

[4] E.H. Lieb, Physica A 140, 240 (1986).

[5] K. Czajka, M.M. Maśka, Physica B, in press.

[6] G.H. Wannier, Phys. Rev. 79, 357 (1950).

[7] See e.g., J.K. Freericks, V. Zlatić, Rev. Mod. Phys. 75, 1333 (2003).

[8] J. Wojtkiewicz, cond-mat/0310043.

[9] M.M. Maśka, K. Czajka, Phys. Status Solidi B 242, 479 (2005); M.M. Maśka, K. Czajka, Physica B 359, 693 (2005).

[10] D.P. Landau, Phys. Rev. B 21, 1285 (1980).

[11] D.P. Landau, K. Binder, Phys. Rev. B 31, 5946 (1985). 